\title{
Outcome of extensive varus and valgus stem alignment in short- stem THA: clinical and radiological analysis using EBRA-FCA
}

\author{
Karl Philipp Kutzner ${ }^{1}$ Tobias Freitag ${ }^{2} \cdot$ Stefanie Donner $^{1} \cdot$ \\ Mark Predrag Kovacevic ${ }^{3} \cdot$ Ralf Bieger $^{2}$
}

Received: 25 July 2016 / Published online: 2 February 2017

(C) The Author(s) 2017. This article is published with open access at Springerlink.com

\begin{abstract}
Introduction The principle of implanting a calcar-guided short stem consists of an individual alignment alongside the medial calcar providing the ability of reconstructing varus and valgus anatomy in a great variety. However, still, there are broad concerns about the safety of extensive varus and valgus positioning in regard to stability, bony alterations, and periprosthetic fractures.

Materials and methods 216 total hip arthroplasties using a calcar-guided short stem (optimys, Mathys Ltd.) in 162 patients were included. Depending on postoperative CCD angle, hips were divided into five groups (A-E). Varus- and valgus tilt and axial subsidence were assessed by "EinzelBild-Roentgen-Analyse"(EBRA-FCA, femoral component analysis) over a 2-year follow-up. The incidence of stressshielding and cortical hypertrophy as well as clinical outcome [Harris Hip Score (HHS)] were reported.
\end{abstract}

Karl Philipp Kutzner

kkutzner@joho.de

Tobias Freitag

Tobias.Freitag@rku.de

Stefanie Donner

sdonner@joho.de

Ralf Bieger

Ralf.Bieger@rku.de

1 Department of Orthopaedic Surgery and Traumatology, St. Josefs Hospital Wiesbaden, Beethovenstr. 20, 65189 Wiesbaden, Germany

2 Department of Orthopaedic Surgery, University of Ulm, Oberer Eselsberg 45, 89081 Ulm, Germany

3 Department of Traumatology, Hand- and Orthopaedic Surgery, HELIOS Dr. Horst Schmidt Clinic Wiesbaden, Ludwig-Erhard-Str. 100, 65199 Wiesbaden, Germany
Results Postoperative CCD angles ranged from $117.9^{\circ}$ to $145.6^{\circ}$ and mean postoperative CCD angles in group A-E were $123.3^{\circ}, 128.0^{\circ}, 132.4^{\circ}, 137.5^{\circ}$, and $142.5^{\circ}$, respectively. After 2 years, the mean varus/valgus tilt was $-0.16^{\circ}$, $0.37^{\circ}, 0.48^{\circ}, 0.01^{\circ}$, and $0.86^{\circ}$, respectively $(p=0.502)$. Axial subsidence after 2 years was $1.20,1.02,1.44,1.50$, and $2.62 \mathrm{~mm}$, respectively $(p=0.043)$. No periprosthetic fractures occurred and none of the stems had to be revised. Rates of stress-shielding and cortical hypertrophy as well as HHS showed no significant difference between the groups. Conclusions Valgus alignment results in increased subsidence but does not affect the clinical outcome. There is no difference in stress shielding and cortical hypertrophy between the groups. The authors recommend long term monitoring of valgus aligned stems.

Keywords Total hip arthroplasty $\cdot$ Short stem $\cdot$ Stem alignment · Optimys · EBRA · Cortical hypertrophy ·

Stress-shielding $\cdot$ Varus $\cdot$ Valgus

\section{Introduction}

In modern primary total hip arthroplasty (THA), short stems are increasingly regarded as implants of first choice [1], especially in young and active patients [2, 3]. All shortstem designs aim at the preservation of proximal bone by reducing stress-shielding due to periprosthetic bone remodelling [4, 5]. However, shortening the femoral stem and reducing the diaphyseal fixation might lead to a possible reduction of stability and lead to changes in the migration pattern [6]. Both, stress-shielding and implant migration, are considered to possibly cause aseptic loosening in cementless THA [5, 7]. 
In the last decade, many different short-stem designs have been introduced $[8,9]$, resulting in a highly heterogenic group. Clinical mid-term results of many designs are encouraging [2, 10-12]. However, also certain designs have already been withdrawn from the market [13].

So-called calcar-guided short stems have been developed to optimally adapt to the anatomy of the proximal femur and to allow a restoration of hip biomechanics [14, 15]. They align themselves alongside the medial cortical bone, sparing the greater trochanter completely [16]. Implantation is done in a "round-the-corner" technique, using individualized levels of osteotomy to align the stem in varus- or valgus position, according to the patient's anatomy $[14,15]$. This results in a broad range of CCD angles to be reconstructed with these types of stems $[15,17]$ with possible extensive varus- and valgus alignments (Fig. 1). Calcar-guided short stems achieve stabilization by metaphyseal anchoring, based on the fit-and-fill principle. However, three-point anchoring is possible in some cases.

To date, little is known about the outcome of extensive varus- or valgus positioning in calcar-guided short-stem THA. There still are broad concerns particularly regarding implant stability. It might have an impact on the rate of axial subsidence as well as on stem tilting. In addition, the influence of different stem alignment on bony alterations like stress-shielding and cortical hypertrophy, as a result of abnormal stress distribution in the loaded proximal femur, to date, is not fully understood. Consequently, it remains unclear that how different stem alignments affect stability and load transfer.

The objective of the present analysis was to address the following research questions:

Does extensive varus- and valgus alignment in calcarguided short-stem THA impact on postoperative varusand valgus tilt and subsidence?

Does different stem alignment affect the incidence of stress-shielding and cortical hypertrophy?

Is there a difference in postoperative functional outcome?

\section{Materials and methods}

In the present retrospective investigation, 216 consecutive hips in 162 patients were included after institutional review board approval (University of Ulm, Germany, 323/13) from an ongoing prospective observational study. Preliminary results of the same study group have already been published involving the same collective of patients in a different context $[18,19]$. Prior to inclusion, written consent to participate has been obtained from all patients. Between 2010 and 2012, 74 women and 88 men were operated using

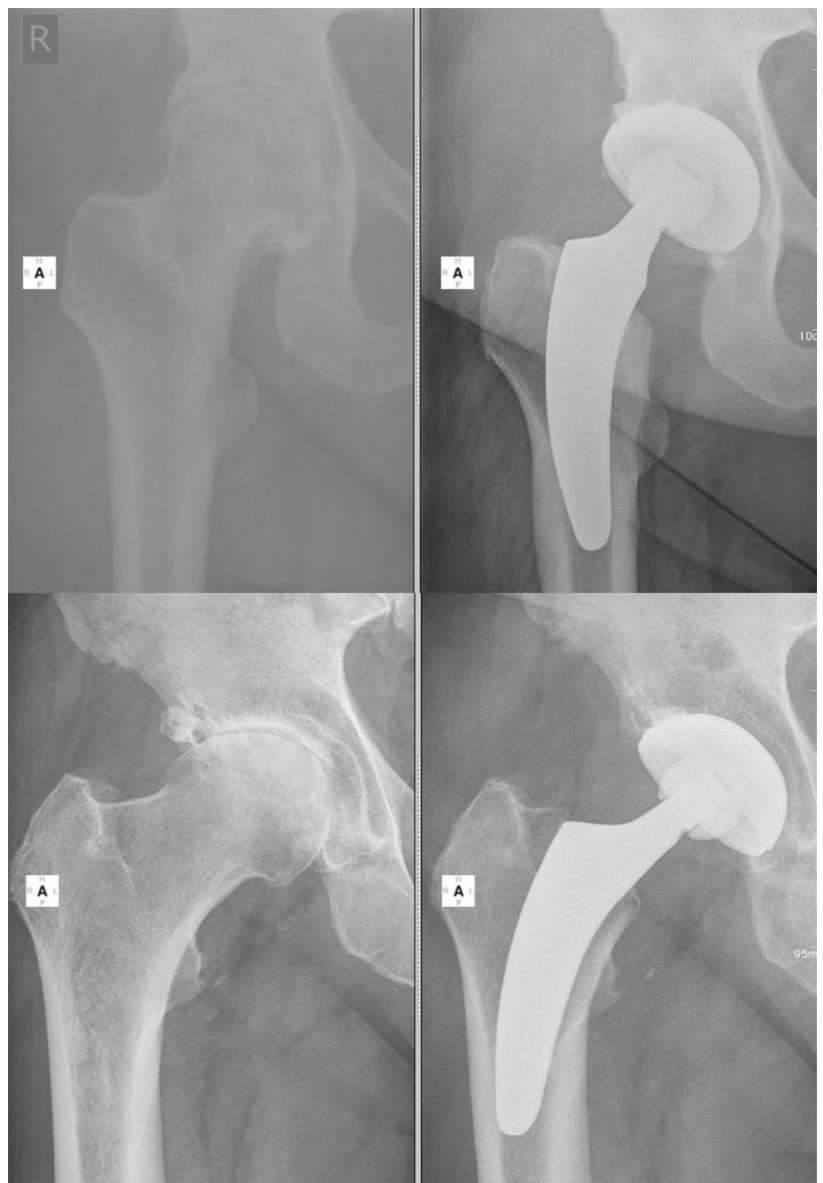

Fig. 1 Possible extensive varus- and valgus stem alignments result in a broad range of CCD angles to be reconstructed with the investigated short stem. Upper row extensive valgus alignment (left preop, right 2-year follow-up); lower row extensive varus alignment (left preop, right 2-year follow-up)

the investigated calcar-guided short-stem. In 54 patients, the treatment was one stage bilaterally; 108 patients were operated unilaterally. The indications for implantation were: $91.7 \%(n=198)$ primary osteoarthrosis, $5.1 \%(n=11)$ femoral head necrosis, $2.3 \%(n=5)$ congenital dysplasia, and $0.9 \%(n=2)$ secondary osteoarthrosis. Mean patient age was 63.0 years (standard deviation (SD) 10.0).

In all patients, the meta-diaphyseal anchoring calcarguided short stem optimys (Mathys Ltd., Bettlach, Switzerland) was implanted as described in Kutzner et al. [18, 19].

The investigated stem was combined with cementless press-fit cups ( $n=177$ Fitmore, Zimmer; $n=39$ RM Pressfit vitamys, Mathys Ltd Bettlach) with a ceramic-polyethylene bearing couple. All surgeries were performed in supine position using a modified, minimally invasive anterolateral approach [20]. Full weight-bearing using two crutches was allowed in all cases immediately after surgery.

All patients underwent pre- and postoperative digital anteroposterior imaging using a standardized technique. 
To produce the deep pelvic radiograph, a positioning splint with $20^{\circ}$ internal rotation of hip joints was used to achieve a standardized and reproducible image during follow-up. $\mathrm{X}$-ray tube was positioned in 1-m distance to the table in perpendicular position. Magnification error was addressed using a ball with known diameter as scaling factor or the known diameter of prosthetic femoral head as an internal reference.

To determine stem tilt and axial stem subsidence, EBRA-FCA (Einzel-Bild-Roentgen-Analyse; University Innsbruck, Austria) was used [21]. A total of 19 reference points were defined on the femoral head (6), the stem (3), the femoral cortex (8), and one at the greater and lesser trochanter, respectively. These reference points define predetermined distances, which are compared by the EBRAFCA software to calculate implant migration. Radiographs with significant positioning artefacts were excluded by the EBRA-FCA software.

For the EBRA-FCA measurements, a series of at least three radiographs was needed. Thus, radiographs during hospital stay as well as after 6, 12, and 24 months were analyzed. For radiological follow-up, including bone resorption and cortical alterations, only radiographs after surgery and after 24 months were considered.

In the postoperative radiograph, CCD angles (between femoral axis and mid-neck axis of the stem) were measured retrospectively, using the digital templating software MediCAD (Hectec, Landshut, Germany; Version 3.5) (Fig. 2). Patients were divided into groups A-E regarding different CCD angles $\left[<124.9^{\circ}(\mathrm{A}) ; 125^{\circ}-129.9^{\circ}(\mathrm{B}) ; 130^{\circ}-134.9^{\circ}\right.$ (C); $135^{\circ}-139.9^{\circ}$ (D); $>140^{\circ}$ (E)]. The rate of stem tilt and axial subsidence, the occurrence of stress-shielding, and cortical hypertrophy, as well as clinical results were analyzed for each group 2 years postoperatively.

Using a modification of the zones described by Gruen [22], bone resorption (stress-shielding) and cortical hypertrophy were analyzed in the standardized radiograph after 2 years (Fig. 3). To detect bone resorption, proximal femoral bone was scanned to find areas with enhanced bonetransparency and thinned or resorbed trabeculae according to the Singh-Index [23]. Grade 1-3 were considered to be stress-shielding. Alterations in periprosthetic cortical bone were analyzed in both radiographs. The increase of cortical width was considered cortical hypertrophy.

In addition, HHS was assessed in all patients after 2 years as well as the rate of periprosthetic fractures.

Mean follow-up time was 2.2 years (range 2.0-3.0 years). Ten hips were either lost to follow up or the EBRA-FCA software failed to accept the radiograph and three patients (4 hips) had deceased unrelated to the operation with prosthesis in situ. In one patient, only a clinical follow-up could be performed. In total, 201 hips in 66 female and 84 male patients fulfilled the inclusion criteria (Table 1).

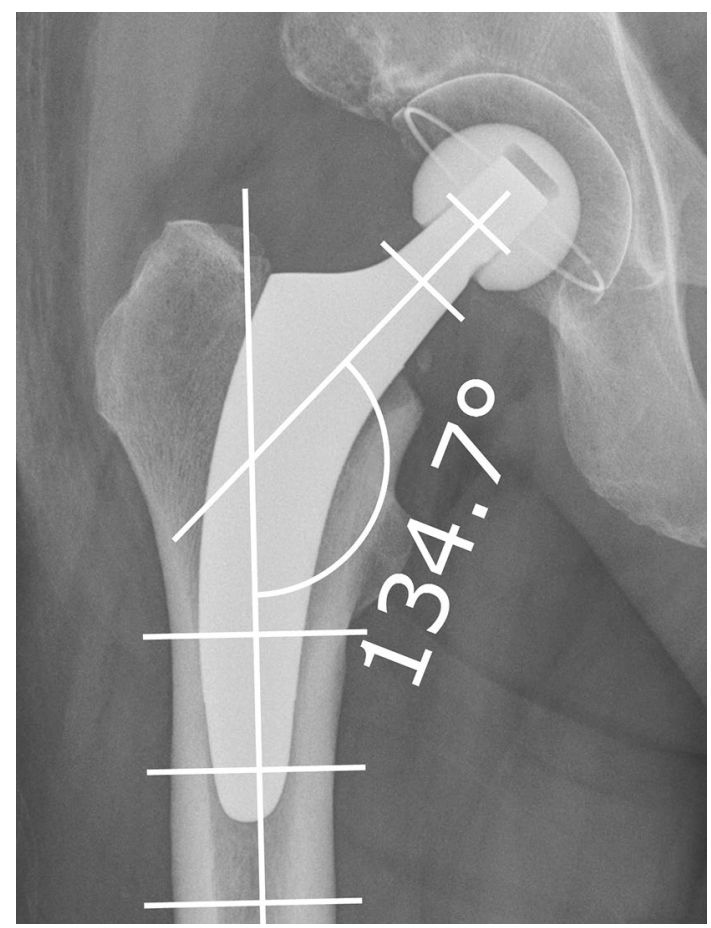

Fig. 2 Measurement of CCD angle (between femoral axis and midneck-axis of the stem) on postoperative radiographs using the digital templating software MediCAD (Hectec, Landshut, Germany)

For statistical evaluation of stem tilt and subsidence, the last follow-up record was used. Differences between groups were examined non-parametrically using Wilcoxon-twosample-tests and Kruskal-Wallis test, respectively (i.e., in case of more than two groups). Correlations between subsidence and CCD postoperative were evaluated using Pearson correlations supplemented by Spearman rank correlations, the latter to evaluate the effect of extreme observations. In addition, regression analyses were carried out relating subsidence to $\mathrm{CCD}$ postop together with age and weight as potential covariates. To this end, robust regression was applied to account for extreme observations. Associations between CCD angle categorizations and discrete variables (such as stress-shielding occurrence) were examined by Chi-square tests.

For statistical significance, a $p$ value of less than 0.05 was considered. The SAS software 9.4 was used for all analyses (SAS Institute, Cary, NC, USA).

\section{Results}

Postoperative range of CCD angles was $117.9^{\circ}-145.6^{\circ}$ with a mean of $132.5^{\circ}$ (SD 4.9). Patients were divided into five groups (A-E) regarding postoperative stem alignment measured by CCD angles. Mean postoperative 
Fig. 3 Analysis of stressshielding and cortical hypertrophy. Left modification of the Gruen-zones; right 2-year follow-up. Stress-shielding (SS) in Gruen-zone 1 and cortical hypertrophy $(\mathrm{CH})$ in Gruenzone 3 and 5

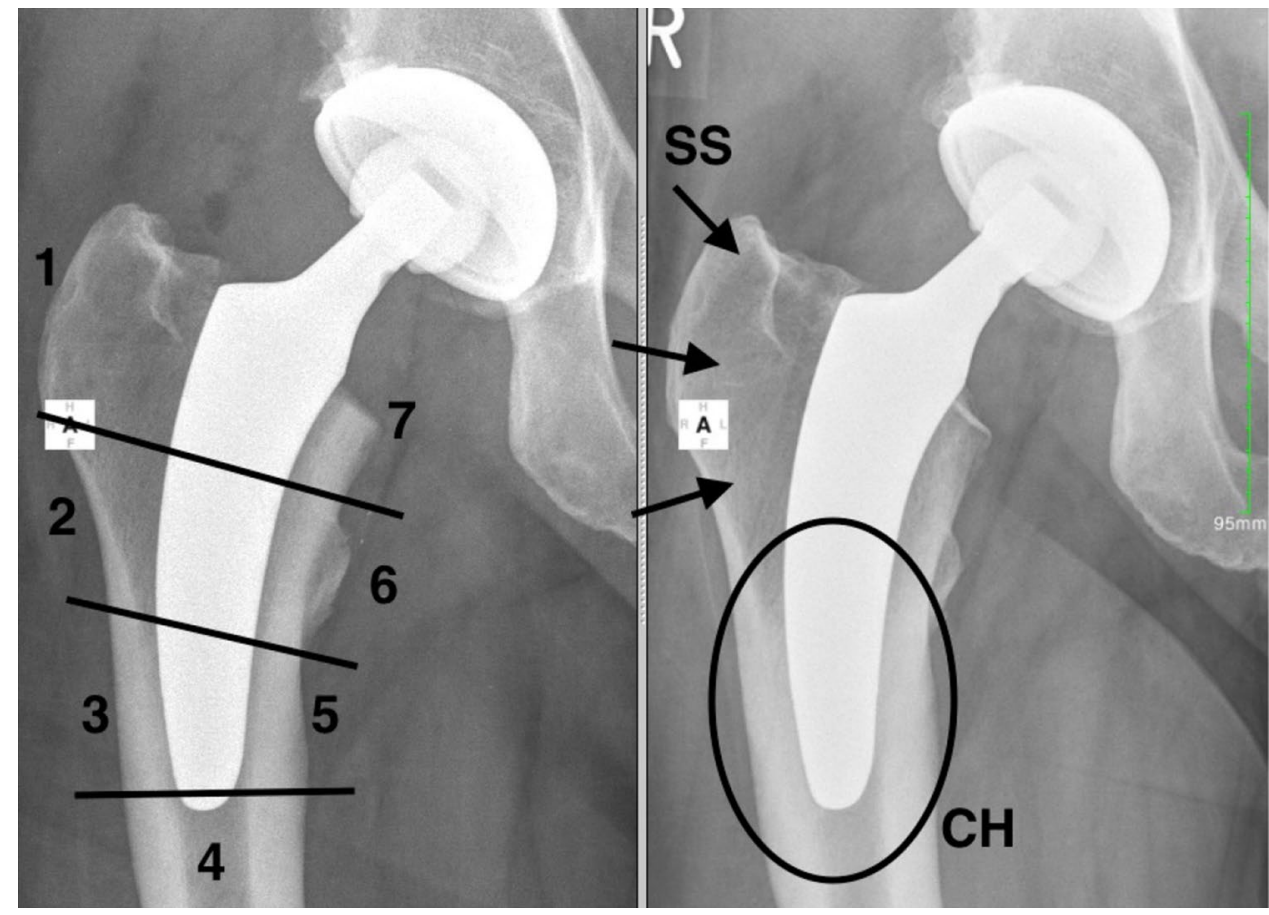

CCD angles for each group were (A) $123.3^{\circ}$ (SD 1.8); (B) $128.0^{\circ}(\mathrm{SD} 1.3)$; (C) $132.4^{\circ}$ (SD 1.4); (D) $137.5^{\circ}$ (SD 1.4); and (E) $142.5^{\circ}$ (SD 2.1), respectively (Table 2).

Mean varus/valgus stem tilt after 2 years was (A) $-0.16^{\circ}$ (SD 3.26); (B) $0.37^{\circ}$ (SD 2.72); (C) $0.48^{\circ}$ (SD 2.28); (D) $0.01^{\circ}$ (SD 2.94); and (E) $0.86^{\circ}$ (SD 2.70), respectively (Table 2 ). There was no evidence for significant differences between the groups $(p=0.502)$. Mean axial subsidence in the investigated groups after 2 years summed up to (A) $1.20 \mathrm{~mm}$ (SD 1.81); (B) $1.02 \mathrm{~mm}$ (SD 1.21); (C) $1.44 \mathrm{~mm}$ (SD 1.34); (D) $1.50 \mathrm{~mm}$ (SD 1.30); and (E) $2.62 \mathrm{~mm}$ (SD 2.17), respectively. Pearson as well as Spearman Correlation showed that the higher the CCD angles are the more pronounced the subsidence was. Regression analysis relating axial subsidence to CCD category together with age and weight as potential covariates accounted for $6.4 \%$ of the variation. Age and weight as covariates turned out to be significant. Axial subsidence increased by about $0.26 \mathrm{~mm}$ for a 10 year age increase and by about $0.13 \mathrm{~mm}$ for a $10 \mathrm{~kg}$ weight increase.

The rate of radiological alterations, such as bone resorption and cortical hypertrophy, is summarized in Table 3. Rates in total are low and there was no statistically significant difference found in any of the categories $(p=0.220$ and $p=0.757$, respectively).

Clinical results, assessed using the HHS, showed no evidence of a difference between different groups after 2 years. Mean HHS in the investigated groups were (A) 96.8 (SD 9.2); (B) 98.1 (SD 4.6); (C) 98.6 (SD 3.5); (D) 97.8 (SD
4.8); and (E) 97.9 (SD 2.5), respectively $(p=0.458)$ (Table 3).

No periprosthetic fractures occurred and none of the stems had to be revised in the observation period.

\section{Discussion}

This study analyzed the outcome of extensive varus- and valgus stem alignment of a calcar-guided short stem regarding implant stability and radiological alterations in a 2-year follow-up. While pronounced femoral varus alignment does not cause increased instability in terms of varus/valgus tilt and axial subsidence, extensive valgus positioning is followed by significantly increased initial subsidence.

The philosophy of calcar-guided short stems emphasizes on the alignment of the stem alongside the medial calcar in different varus- and valgus position, according to the patient's anatomy, using individual levels of neck resection [14, 15]. In a comparison of a modular short stem and a conventional straight stem, Schmidutz et al. found a significantly wider range of stem alignment for the short stem $\left(6.2^{\circ}\right.$ varus to $8.8^{\circ}$ valgus $)$ than for the conventional stem $\left(2.6^{\circ}\right.$ varus to $3.3^{\circ}$ valgus $)$ [17]. In $24 \%$, stems were implanted in pronounced varus position; in $18 \%$, they were placed in pronounced valgus position [17]. The present collective confirms a broad range of postoperative CCD angles using the investigated implant (Table 1). To position the stem individually, the level of neck resection has to be adapted accordingly. A high resection leads the stem 
Table 1 Patient's demographics

\begin{tabular}{|c|c|c|c|c|c|}
\hline \multirow[t]{2}{*}{ CCD category } & \multicolumn{5}{|c|}{ Demographics } \\
\hline & Age & Weight & BMI & Gender $(\mathrm{w} / \mathrm{m})$ & $\begin{array}{l}\text { Uni- } \\
\text { lateral/ } \\
\text { bilateral }\end{array}$ \\
\hline \multicolumn{6}{|l|}{ A } \\
\hline$n$ & 14 & & & $5 / 9$ & $10 / 4$ \\
\hline Mean (SD) & $65.2(12.5)$ & 80.7 (16.7) & $27.6(5.9)$ & & \\
\hline $95 \% \mathrm{CI}$ & $58.0,72.4$ & $71.1,90.4$ & $24.1,31.0$ & & \\
\hline Median & 69.6 & 82 & 27 & & \\
\hline Range & $33-80$ & $53-111$ & $19-40$ & & \\
\hline \multicolumn{6}{|c|}{$\mathrm{B}$} \\
\hline$n$ & 45 & & & $21 / 24$ & $29 / 16$ \\
\hline Mean (SD) & $62.4(9.9)$ & $80.7(12.4)$ & $27.1(4.4)$ & & \\
\hline $95 \% \mathrm{CI}$ & $59.5,65.4$ & $77.0,84.4$ & $25.8,28.4$ & & \\
\hline Median & 62.9 & 81 & 27 & & \\
\hline Range & $40-81$ & $57-115$ & $21-40$ & & \\
\hline \multicolumn{6}{|c|}{$\mathrm{C}$} \\
\hline$n$ & 87 & & & $45 / 42$ & $43 / 44$ \\
\hline Mean (SD) & $64.0(8.9)$ & $82.1(16.1)$ & $27.6(4.4)$ & & \\
\hline $95 \% \mathrm{CI}$ & $62.1,65.9$ & $78.7,85.6$ & $26.6,28.5$ & & \\
\hline Median & 63.4 & 80 & 27 & & \\
\hline Range & $36-87$ & $55-153$ & $21-42$ & & \\
\hline \multicolumn{6}{|c|}{$\mathrm{D}$} \\
\hline$n$ & 42 & & & $14 / 28$ & $15 / 27$ \\
\hline Mean (SD) & $61.0(9.1)$ & $89.5(20.3)$ & $29.5(6.4)$ & & \\
\hline $95 \% \mathrm{CI}$ & $58.2,63.9$ & $83.1,95.8$ & $27.5,31.5$ & & \\
\hline Median & 60.2 & 85.5 & 27 & & \\
\hline Range & $36-77$ & $50-140$ & $20-45$ & & \\
\hline \multicolumn{6}{|c|}{$\mathrm{E}$} \\
\hline$n$ & 13 & & & $2 / 11$ & $2 / 11$ \\
\hline Mean (SD) & $62.7(8.0)$ & $87.8(17.8)$ & $27.2(4.6)$ & & \\
\hline $95 \% \mathrm{CI}$ & $57.9,67.6$ & $77.0,98.5$ & $24.4,29.9$ & & \\
\hline Median & 61 & 83 & 27 & & \\
\hline Range & $54-77$ & $60-125$ & $21-37$ & & \\
\hline \multicolumn{6}{|l|}{ Total } \\
\hline$n$ & 201 & & & $87 / 114$ & $51 / 99$ \\
\hline Mean (SD) & $63.0(9.4)$ & $83.6(16.7)$ & $27.8(5.02)$ & & \\
\hline $95 \% \mathrm{CI}$ & $61.7,64.3$ & $81.3,85.9$ & $21.7,28.5$ & & \\
\hline Median & 63.0 & 82.0 & 27.0 & & \\
\hline Range & $33-87$ & $50-153$ & $19-45$ & & \\
\hline
\end{tabular}

$n$ number of cases, $S D$ standard deviation, $95 \%$ CI 95\% confidence interval

alongside the calcar in a varus position; a valgus position is achieved by deep resection [14, 15]. However, the effect of extensive varus- and valgus alignment on implant migration following short calcar-guided stems has not been investigated. Aseptic loosening is considered to be the most common reason for implant revision [24]. In this context, primary stability, as well as the design specific potential to maintain proximal femoral bone stock is of great importance to predict implant failure [6]. For fixation of metaphyseal anchoring short stems, a sufficient cortical ring is recommended [5]. In varus anatomy, given a high neck resection, ring-fixation is accentuated. However, in valgus anatomy, given the need of a low resection level to place the stem in the intended valgus position, the ring-fixation might be compromised [5] with subsequent varus- or valgus tilt and axial subsidence after full weight bearing. Therefore, in addition, sufficient contact of the tip of the stem to the lateral cortex in regard to achieving a classical 
three-point anchoring is of great importance (Fig. 4). Again, in pronounced varus stem alignment, contact of the tip of the stem to the lateral cortex is commonly achieved.
In valgus position, however, a missing cortical contact of the tip can be frequently observed, particularly in cases of "undersizing" (Fig. 4). Hence, the operating surgeon should
Table 2 Rate of axial valgus tilt $\left(^{\circ}\right)$ measured in different CCD categories (A-E)

Table 3 Stress-shielding, cortical hypertrophy, and Harris Hip Score analyzed in different CCD categories (A-E) subsidence ( $\mathrm{mm}$ ) and varus- $/$

\begin{tabular}{|c|c|c|c|c|c|c|c|c|c|c|c|}
\hline \multirow[t]{2}{*}{ CCD category } & \multirow[t]{2}{*}{$n$} & \multicolumn{5}{|c|}{ Axial subsidence } & \multicolumn{5}{|c|}{ Varus/valgus tilt } \\
\hline & & Mean & SD & Median & \multicolumn{2}{|c|}{$95 \% \mathrm{CI}$} & Mean & SD & Median & \multicolumn{2}{|l|}{$95 \% \mathrm{CI}$} \\
\hline $\mathrm{A}(7.0 \%)$ & 14 & -1.20 & 1.81 & -0.65 & -2.25 & -0.15 & -0.16 & 3.26 & -0.15 & -2.05 & 1.7 \\
\hline B $(22.4 \%)$ & 45 & -1.02 & 1.21 & -0.70 & -1.38 & -0.65 & 0.37 & 2.72 & -0.20 & -0.44 & 1.19 \\
\hline C (43.3\%) & 87 & -1.44 & 1.34 & -1.20 & -1.72 & -1.15 & 0.48 & 2.28 & 0.50 & -0.01 & 0.96 \\
\hline D (20.9\%) & 42 & -1.50 & 1.30 & -1.35 & -1.91 & -1.10 & 0.01 & 2.94 & -0.20 & -0.90 & 0.93 \\
\hline $\mathrm{E}(6.5 \%)$ & 13 & -2.62 & 2.17 & -2.20 & -3.93 & -1.30 & 0.86 & 2.70 & 0.80 & -0.77 & 2.4 \\
\hline Total $(100.0 \%)$ & 201 & -1.42 & 144 & -1.20 & -1.62 & -1.22 & 0.34 & 2.61 & 0.20 & -0.03 & 0.7 \\
\hline & & \multicolumn{5}{|c|}{$\begin{array}{l}\text { Kruskal-Wal- } \\
\quad \text { lis test }(d f=4): p=0.043\end{array}$} & \multicolumn{5}{|c|}{$\begin{array}{l}\text { Kruskal-Wallis } \\
\quad \text { test }(d f=4): p=0.502\end{array}$} \\
\hline
\end{tabular}

\begin{tabular}{|c|c|c|c|c|c|c|c|c|}
\hline \multirow[t]{2}{*}{$\overline{C C D}$ category } & \multirow[t]{2}{*}{$n$} & \multirow[t]{2}{*}{ Stress-shielding } & \multirow[t]{2}{*}{ Cortical hypertrophy } & \multicolumn{5}{|c|}{ Harris Hip Score } \\
\hline & & & & Mean & Stdev & Median & $95 \% \mathrm{C}$ & \\
\hline $\mathrm{A}(7.0 \%)$ & 14 & $0(0.0 \%)$ & $0(0.0 \%)$ & 96.79 & 9.23 & 100 & 91.45 & 102.10 \\
\hline B (22.4\%) & 45 & $1(2.2 \%)$ & $2(4.4 \%)$ & 98.09 & 4.57 & 100 & 96.72 & 99.46 \\
\hline $\mathrm{C}(43.3 \%)$ & 87 & $4(4.6 \%)$ & $3(3.4 \%)$ & 98.60 & 3.48 & 100 & 97.86 & 99.34 \\
\hline D (20.9\%) & 42 & $1(2.4 \%)$ & $3(7.1 \%)$ & 97.83 & 4.81 & 100 & 96.33 & 99.33 \\
\hline $\mathrm{E}(6.5 \%)$ & 13 & $2(15.4 \%)$ & $1(7.7 \%)$ & 97.92 & 2.53 & 99 & 96.39 & 99.45 \\
\hline Total $(100.0 \%)$ & 201 & $8(4.0 \%)$ & $9(4.5 \%)$ & 98.15 & 4.55 & 100 & 97.52 & 98.79 \\
\hline & & $\begin{array}{l}\text { Chi-square- } \\
\text { statistics }(d f=4) \\
p=0.220\end{array}$ & $\begin{array}{l}\text { Chi-square-statistics } \\
(d f=4) p=0.757\end{array}$ & \multicolumn{5}{|c|}{$\begin{array}{l}\text { Kruskal-Wal- } \\
\quad \text { lis test }(d f=4): p=0.458\end{array}$} \\
\hline
\end{tabular}

Fig. 4 "Undersizing" accompanied with a lack of contact to the lateral cortex, especially in valgus hips, might support initial instability with subsequent implant micromovement
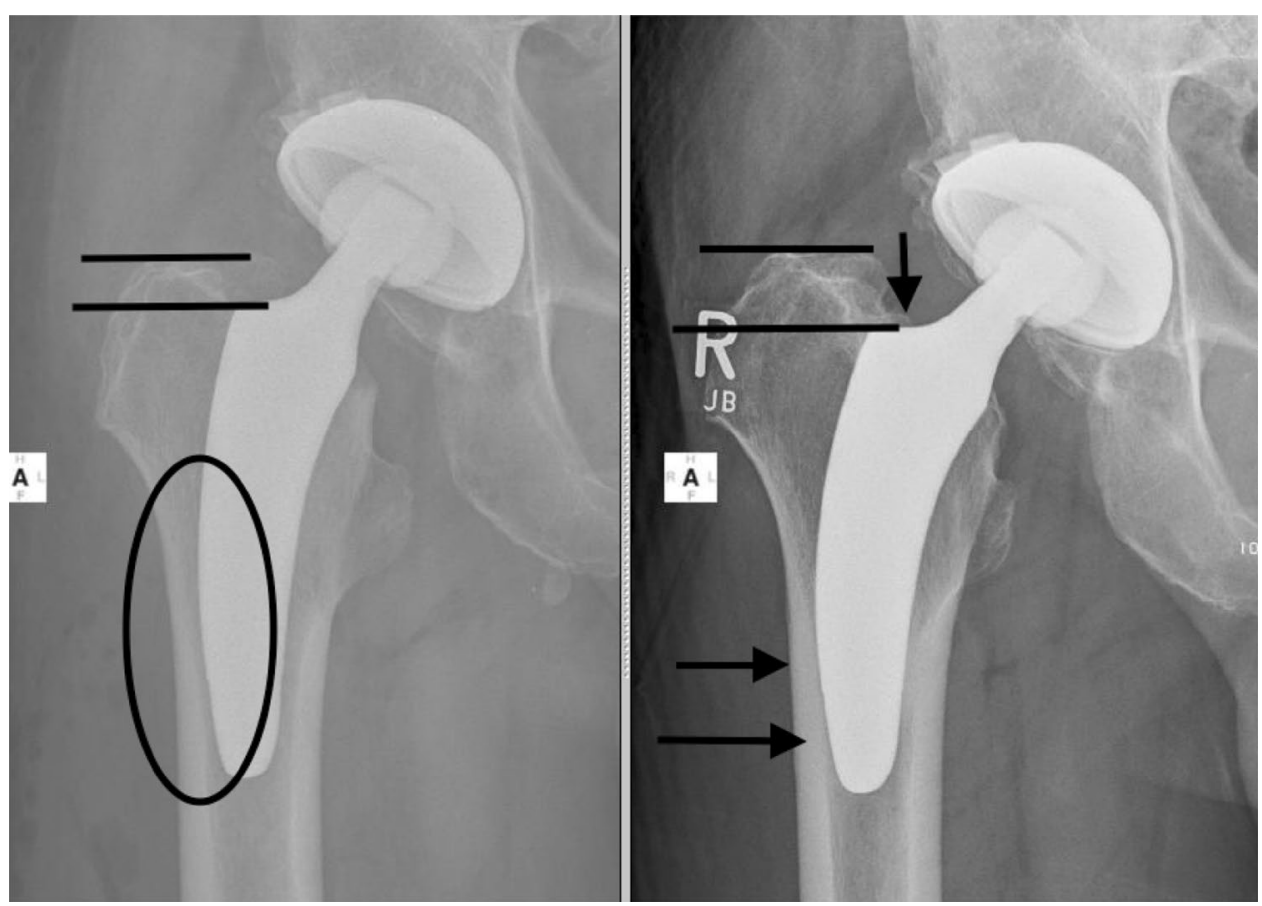
be aware, that "undersizing" accompanied with a lack of contact to the lateral cortex, especially in valgus hips, might support initial instability with subsequent implant micromovement. Due to the different implant design of the investigated stem, in contrast to the Metha stem, in those cases, a resection to the level of the fossa piriformis should be pursued, to implant the adequate size of the stem and avoid "undersizing". Earlier published results on the migration pattern of the investigated short stem showed a significant influence of body weight on the rate of early axial subsidence [19]. The regression analysis relating axial subsidence to CCD categories confirms the impact of heavy weight accordingly in the present investigation. However, the model accounted for only $6.4 \%$ of the variation.

Furthermore, it remains unclear how different resection heights and stem alignments affect load transfer and stressshielding patterns, which are, besides micromovement of the implant, also generally thought to have a considerable effect on aseptic loosening [5]. Lerch et al. found no correlation between stem alignment, stem size, or resection level with changes in bone mineral density (BMD) in a recent investigation of the Metha short stem (Aesculap, Tuttlingen, Germany) using dual-energy X-ray absorptiometry (DEXA) [25]. However, the small size of the study population might not allow valid conclusions. Floerkemeier et al., in a biomechanical study using strain gauges on synthetic composite femora, measuring strain patterns of three different resection levels after implanting also the Metha short stem (Aesculap, Tuttlingen, Germany), found a decrease in strain in the region of the greater trochanter by 30,32 , and $52 \%$ for the high-, medium-, and low-neck resection level, respectively [5]. This corresponds to the results of this study, with stems aligned in extensive valgus position showing slightly enhanced stress-shielding in Gruen-zone 1 , compared to implants in varus position, although not being statistically significant. In Gruen-zone 7, the medial calcar cortex, the biomechanical measurements resulted in slightly reduced strains for the low- and medium-resection level, while there was a strain increase of $43 \%$ for the highresection level [5]. Again, this study confirms these findings, showing a slightly pronounced bone resorption on the medial part of the neck in valgus hips with low resection level, compared to varus hips with high-resection level, however, without any statistical significance.

Aligning the stem in an extensive varus position results in an increase in offset and subsequent enhanced lever-arm compared to the extensive valgus alignment, in turn, leading to an increased strain in the medial part of the proximal femur and the region around the distal tip of the stem [5]. Given increased forces, especially on the medial cortical bone, with some stem designs, a higher risk of intraoperative and postoperative periprosthetic fractures has been reported [9]. However, after 2 years in the present investigation, no periprosthetic fractures were observed. Theoretically, abnormal load distribution might lead to cortical hypertrophy in Gruen-zones 3 and 5 with possible subsequent thigh pain $[10,26]$. In a recent study, analyzing the Fitmore stem (Zimmer, Warsaw, IN, USA), cortical hypertrophy was observed in $63 \%$ of hips accompanied with a low rate of thigh pain, implying distal loading and proximal stress bypass [27]. In contrast, the optimys stem is designed with a polished tip to retain strain peaks in the distal part and, therefore, avoid cortical reactions. The overall incidence after a follow-up of 2 years has been shown to be below $5 \%$ without any appearance of thigh pain or other clinical consequences [28]. A slight emphasis regarding cortical hypertrophy could be found for valgus hips, however, not being statistically significant. The small number of cortical hypertrophy in the present study does not allow valid conclusions regarding the impact of different extensive stem alignment.

This study has several limitations. First to be mentioned is the short follow-up of 2 years. Although only long-term results should be considered valid, the initial evaluation of bony alterations is necessary to identify undesirable results [29]. Early migration analysis may allow a prediction of implant survival [7] and the reaction of environmental bone in the early stage may help to predict the long-term outcome. Second, RSA provides higher accuracy in comparison to the EBRA-FCA method used in this study. The computer-assisted EBRA-FCA system was evaluated to be able to detect stem subsidence of $\pm 1 \mathrm{~mm}$ and varus/valgus tilting of $\pm 0.4^{\circ}$ given a specificity of $100 \%$ and sensitivity of $78 \%$ [21]. However, the migration pattern after 2 years has been established in several studies using EBRA-FCA providing a reference to long-term survival [7, 30]. Furthermore, measuring the $\mathrm{CCD}$ angles in $2 \mathrm{D}$ radiographs lacks some accuracy compared to 3D imaging. However, radiographs were done using a standardized protocol to reduce a possible bias.

\section{Conclusions}

The results confirm a wide range of CCD angles to be adequately reconstructed using the investigated calcar-guided short stem. After 2 years, extensive varus positioning does not cause increased instability in terms of varus/valgus tilt and axial subsidence suggesting no restrictions regarding indications. Extensive valgus positioning is followed by significantly increased subsidence without any clinical correlation. Undersizing the stem providing insufficient cortical contact could be identified as the main cause leading to axial subsidence, especially in extensive valgus alignments. The overall rate of stress-shielding and cortical hypertrophy is low without any clinical consequences. Short-term 
clinical results in all groups are encouraging. Further monitoring, especially of the valgus hips, is mandatory.

Acknowledgements We thank Dominik Pfluger (numerics data $\mathrm{GmbH}$ ) for supporting statistical analysis. Mathys Ltd. (Switzerland) funded the costs for additional radiographs in radiological follow-up examinations. No other fundings were received.

\section{Compliance with Ethical Standards}

All procedures performed in studies involving human participants were in accordance with the ethical standards of the institutional research committee and with the 1964 Helsinki declaration and its later amendments or comparable ethical standards. Institutional review board approval (University of Ulm, Germany, 323/13) has been obtained.

Conflict of interest The authors declare that they have no conflict of interest.

Open Access This article is distributed under the terms of the Creative Commons Attribution 4.0 International License (http:// creativecommons.org/licenses/by/4.0/), which permits unrestricted use, distribution, and reproduction in any medium, provided you give appropriate credit to the original author(s) and the source, provide a link to the Creative Commons license, and indicate if changes were made.

\section{References}

1. Zeh A, Radetzki F, Diers V et al (2011) Is there an increased stem migration or compromised osteointegration of the Mayo short-stemmed prosthesis following cerclage wiring of an intrasurgical periprosthetic fracture? Arch Orthop Trauma Surg 131:1717-1722. doi:10.1007/s00402-011-1342-1

2. Ettinger M, Ettinger P, Lerch $M$ et al (2011) The NANOS short stem in total hip arthroplasty: a mid term follow-up. Hip Int 21:583-586. doi:10.5301/HIP.2011.8658

3. Gulow J, Scholz R, Freiherr von Salis-Soglio G (2007) Shortstemmed endoprostheses in total hip arthroplasty. Der Orthopäde 36:353-359. doi:10.1007/s00132-007-1071-x

4. Aamodt A, Lund-Larsen J, Eine J et al (2001) Changes in proximal femoral strain after insertion of uncemented standard and customised femoral stems. An experimental study in human femora. J Bone Joint Surg Br 83:921-929

5. Floerkemeier T, Gronewold J, Berner S et al (2013) The influence of resection height on proximal femoral strain patterns after Metha short stem hip arthroplasty: an experimental study on composite femora. Int Orthop 37:369-377. doi:10.1007/ s00264-012-1725-0

6. Bieger R, Ignatius A, Decking R et al (2012) Primary stability and strain distribution of cementless hip stems as a function of implant design. Clin Biomech (Bristol Avon) 27:158-164. doi:10.1016/j.clinbiomech.2011.08.004

7. Krismer M, Biedermann R, Stöckl B et al (1999) The prediction of failure of the stem in THR by measurement of early migration using EBRA-FCA. Einzel-Bild-Roentgen-Analyse-femoral component analysis. J Bone Joint Surg Br 81:273-280

8. Khanuja HS, Banerjee S, Jain D et al (2014) Short bone-conserving stems in cementless hip arthroplasty. J Bone Joint Surg Am 96:1742-1752. doi:10.2106/JBJS.M.00780

9. Banerjee S, Pivec R, Issa K et al (2013) Outcomes of short stems in total hip arthroplasty. Orthopedics 36:700-707. doi:10.3928/01477447-20130821-06
10. Gustke K (2012) Short stems for total hip arthroplasty: initial experience with the Fitmore stem. J Bone Joint Surg Br 94:4751. doi:10.1302/0301-620X.94B11.30677

11. von Lewinski G, Floerkemeier T (2015) 10-year experience with short stem total hip arthroplasty. Orthopedics 38:S51-S56. doi:10.3928/01477447-20150215-57

12. Kovacevic MP, Pfeil J, Kutzner KP (2014) Implantation of a new short stem in simultaneous bilateral hip arthroplasty-a prospective study on clinical and radiographic data of 54 consecutive patients. OUP 10:456-461. doi:10.3238/oup.2014.0456-0461

13. Jerosch J (2012) Kurzschaft ist nicht gleich Kurzschaft-Eine Klassifikation der Kurzschaftprothesen. OUP. doi:10.3238/ oup.2012.0304-0312

14. Jerosch J, Grasselli C, Kothny PC et al (2012) Reproduction of the anatomy (offset, $\mathrm{CCD}$, leg length) with a modern short stem hip design - a radiological study. Z Orthop Unfall 150:20-26. doi:10.1055/s-0030-1270965

15. Kutzner KP, Kovacevic MP, Roeder C et al (2014) Reconstruction of femoro-acetabular offsets using a short-stem. Int Orthop. doi:10.1007/s00264-014-2632-3

16. Pfeil J (2014) Comment je pose la prothèse optimys. Maitrise Orthopedique 236:8

17. Schmidutz F, Beirer M, Weber P et al (2012) Biomechanical reconstruction of the hip: comparison between modular shortstem hip arthroplasty and conventional total hip arthroplasty. Int Orthop 36:1341-1347. doi:10.1007/s00264-011-1477-2

18. Kutzner KP, Kovacevic MP, Freitag T et al (2016) Influence of patient-related characteristics on early migration in calcar-guided short-stem total hip arthroplasty: a 2-year migration analysis using EBRA-FCA. J Orthop Surg Res 11:29. doi:10.1186/ s13018-016-0363-4

19. Kutzner KP, Freitag T, Kovacevis MP et al (2016) One-stage bilateral versus unilateral short-stem total hip arthroplasty: comparison of migration patterns using "Ein-Bild-RoentgenAnalysis Femoral-Component-Analysis" Int Orthop 41:61-66. doi:10.1007/s00264-016-3184-5

20. Pfeil J (2010) Minimally invasive surgery in total hip arthroplasty [English]. Springer, Berlin

21. Biedermann R, Krismer M, Stöckl B et al (1999) Accuracy of EBRA-FCA in the measurement of migration of femoral components of total hip replacement. Einzel-Bild-Röntgen-Analysefemoral component analysis. J Bone Joint Surg Br 81:266-272

22. Gruen TA, McNeice GM, Amstutz HC (1979) "Modes of failure" of cemented stem-type femoral components: a radiographic analysis of loosening. Clin Orthop Relat Res 141:17-27

23. Singh M, Nagrath AR, Maini PS (1970) Changes in trabecular pattern of the upper end of the femur as an index of osteoporosis. J Bone Joint Surg Am 52:457-467

24. Kärrholm J (2012) Radiostereometric analysis of early implant migration - a valuable tool to ensure proper introduction of new implants. Acta Orthop 83:551-552. doi:10.3109/17453674.2012. 745352

25. Lerch M, von der Haar-Tran A, Windhagen $\mathrm{H}$ et al (2012) Bone remodelling around the Metha short stem in total hip arthroplasty: a prospective dual-energy X-ray absorptiometry study. Int Orthop 36:533-538. doi:10.1007/s00264-011-1361-0

26. Ritter MA, Fechtman RW (1988) Distal cortical hypertrophy following total hip arthroplasty. J Arthroplasty 3:117-121

27. Maier MW, Streit MR, Innmann MM et al (2015) Cortical hypertrophy with a short, curved uncemented hip stem does not have any clinical impact during early follow-up. BMC Musculoskelet Disord 16:371. doi:10.1186/s12891-015-0830-9

28. Kutzner KP, Pfeil D, Kovacevic MP et al (2016) Radiographic alterations in short-stem total hip arthroplasty: a 2-year follow-up study of 216 cases. Hip Int 26:278-283. doi:10.5301/ hipint.5000339 
29. Baad-Hansen T, Kold S, Olsen N et al (2011) Excessive distal migration of fiber-mesh coated femoral stems. Acta Orthop 82:308-314. doi:10.3109/17453674.2011.574562

30. Freitag T, Kappe T, Fuchs M et al (2014) Migration pattern of a femoral short-stem prosthesis: a 2-year EBRA-FCA-study.
Arch Orthop Trauma Surg 134:1003-1008. doi:10.1007/ s00402-014-1984-X 